\title{
EXPERIENCIAS DE GESTIÓN DE LOS PROCESOS DE PRESERVACIÓN DIGITAL A PARTIR DEL MODELO OAIS EN REPOSITORIOS INSTITUCIONALES
}

\author{
Jaider Ochoa-Gutiérrez* \\ Escuela Interamericana de Bibliotecología. Universidad de Antioquia. \\ Andrés Sáenz-Giraldo** \\ Escuela Interamericana de Bibliotecología. Universidad de Antioquia. \\ Tatiana Tirado-Tamayo ${ }^{* * *}$ \\ Escuela Interamericana de Bibliotecología. Universidad de Antioquia.
}

\begin{abstract}
Resumen: Los repositorios institucionales representan la denominada Vía Verde del movimiento Acceso Abierto, facilitando el acceso a la información y la gestión de la memoria científica de las universidades y centros de investigación. En ese sentido, no solo debe optimizarse su funcionamiento en términos de garantizar la organización y recuperación de los documentos sino también, los procesos de interoperabilidad y preservación digital. Es aquí donde el Open Archival Information System (OAIS) ha tomado lugar como modelo y estándar que orienta y optimiza los procesos de cara a la preservación digital. A partir de lo anterior, este artículo da cuenta de los resultados de una investigación documental sobre el uso del modelo OAIS en repositorios institucionales, para ello se presenta la metodología de búsqueda desarrollada y el análisis de los factores a considerar en la gestión de los repositorios. Los resultados recopilan experiencias y categorías de implementación, principios básicos y esquemas de funcionamiento para atender condiciones de integralidad, interoperabilidad y facilidad en la recuperación y preservación de la memoria científica y académica de las instituciones. Principalmente se puede evidenciar una tendencia a modelos y arquitecturas distribuidas, mayor cantidad de experiencias en Europa y Estados Unidos y el uso de sistemas y software como DSpace, Lockss y Archivematica.
\end{abstract}

Palabras clave: Acceso abierto; repositorio institucional; modelo OAIS; preservación digital.

Title: EXPERIENCES IN MANAGEMENT OF DIGITAL PRESERVATION PROCESSES FROM THE OAIS MODEL IN INSTITUTIONAL REPOSITORIES.

Abstract: The institutional repositories represent the so-called Greenroad of the Open Access movement, facilitating access to information and the management of the scientific memory of universities and research centers. In this sense, its operation should not only be optimized in terms of guaranteeing the organization and retrieval of documents, but also optimizing the processes of interoperability and digital preservation. It is here where the Open Archival Information System (OAIS) has taken place as the standard that guides and optimizes processes for digital preservation. Based on the above, this article reports the results of documentary research on the use of the OAIS model in institutional repositories; for this purpose the developed search methodology and the analysis of the factors to be considered in the management of the repositories are presented. The results compile experiences and implementation categories, basic principles, and operating schemes to meet conditions of comprehensiveness, interoperability, and ease in the recovery and preservation of the scientific and academic memory of the institutions. Mainly a trend towards distributed models and architectures, a greater number of experiences in Europe and the United States and the use of systems and software such as DSpace, Lockss, and Archivematica can be evidenced. Keywords: Open access; institutional repository; OAIS model; digital preservation.

Copyright: (C) 2021 Servicio de Publicaciones de la Universidad de Murcia (Spain). Este es un artículo de acceso abierto distribuido bajo los términos de la licencia Creative Commons Reconocimiento 4.0 Internacional (CC BY $4.0)$.

\section{INTRODUCCIÓN}

El movimiento de Acceso Abierto busca la eliminación tanto de barreras económicas como de aquellas derivadas de los derechos de explotación que limitan la difusión, el acceso y la reutilización de recursos resultado de la producción científica y académica (Vicente, Melero y Abadal, 2014). Originalmente, se manifiesta a partir de dos rutas o modalidades: la ruta dorada, la cual promulga la publicación en revistas en acceso abierto; y la ruta verde, cuya máxima expresión son los repositorios digitales, ya sean institucionales o temáticos, los cuales permiten incorporar, preservar,

\footnotetext{
*jaider.ochoa@udea.edu.co

***inaldo.saenz@udea.edu.co

*** tatiana.tirado10367@udea.edu.co

Recibido: 16-05-2020; 2 ${ }^{\mathrm{a}}$ versión: 15-02-2021; aceptado: 22-02-2021.
}

OCHOA GUTIÉRREZ, J.; SÁENZ GIRALDO, A. y TIRADO TAMAYO, T. Experiencias de gestión de los procesos de preservación digital a partir del modelo OAIS en repositorios institucionales. Anales de Documentación, 2021, vol. 24, $\mathrm{n}^{\circ} 1$. Disponible en: http://dx.doi.org/10.6018/analesdoc.428141. 
consultar y dar soporte a la gestión y difusión de los recursos digitales creados por la propia institución o disciplina temática, mediante una adecuada clasificación de sus recursos a través de metadatos (Lynch, 2003).

Para el caso de los repositorios, estos se caracterizan por ser acumulativos, perpetuos, de libre acceso e interoperables (Crow, 2002); por ello, en un ámbito institucional académico, se convierten en herramientas necesarias para la disposición de la producción científica y académica de cara a los retos que presenta la digitalización en la actualidad y de la lucha constante por promover el libre acceso a la información para superar las barreras impuestas por el mercado editorial científico.

Uno de los retos fundamentales respecto a la gestión de repositorios institucionales obedece a la preservación digital, pues la documentación disponible en estos representa la memoria institucional la cual debe mantenerse accesible en el tiempo. Para ello, propuestas como el modelo de referencia OAIS (Sistema de Información de Archivo Abierto), buscan definir los procesos necesarios para la preservación y acceso a la información de manera efectiva y a largo plazo (Cruz Mundet y Díez Carrera, 2016). Sin embargo, este modelo ha sido utilizado principalmente en repositorios de archivo asociados a sistemas de gestión de documentos, pues tal como lo menciona Raventós: "El modelo de referencia Open Archival Information System (OAIS) se ha erigido como el modelo sobre el cual muchos repositorios de archivo para la conservación a largo plazo han basado su arquitectura" (Raventós, 2010, p. 7); por lo que representa un reto su implementación en repositorios institucionales de cara no solo a facilitar el acceso a la información científica y académica, sino a garantizar su preservación en el tiempo.

Este artículo tiene como propósito presentar los resultados de una investigación documental sobre el uso del modelo OAIS en repositorios institucionales, para ello se presenta la metodología de búsqueda que se desarrolló, teniendo en cuenta el análisis de los procesos y factores a considerar en la gestión de los repositorios. Los resultados evidencian, principalmente, poca información del uso del modelo en repositorios institucionales. Sin embargo, se logró la recopilación de experiencias y categorías de implementación, principios básicos y esquemas de funcionamiento para atender condiciones de integralidad, interoperabilidad y facilidad en la recuperación y preservación de la memoria científica y académica de las instituciones. En este sentido, se puede evidenciar una tendencia a modelos y arquitecturas distribuidas, las cuales permiten la disminución de costos y el desarrollo de acuerdos para mejorar la interoperabilidad. Así mismo, se identifica mayor cantidad de experiencias en Europa y Estados Unidos y el uso de sistemas y software como DSpace, Lockss y Archivematica.

\section{OBJETIVOS Y METODOLOGÍA}

Este trabajo tiene como objetivo analizar el panorama de experiencias, recomendaciones y recursos para la preservación digital en repositorios institucionales a partir del uso del modelo OAIS. Para ello se realiza una investigación documental, acorde con las recomendaciones propuestas por Cochrane (Higgins y Thomas, 2019) para realizar revisiones sistemáticas, permitiendo no solo dar cuenta de las experiencias sino analizar la importancia de los procesos de preservación digital y los requisitos necesarios para el diseño de estrategias.

Para la búsqueda de información se restringió a un periodo entre 2009 y 2020, considerando la importancia de identificar los últimos desarrollos en la temática definida. Las fuentes de información consideradas fueron, en primera instancia, Scopus, Web of Science, reconocidas como bases de datos referenciales de gran aceptación en el ámbito académico y con una cobertura mayor para la producción académica en inglés; Google Scholar y Lens.org, fuentes de información en abierto de gran cobertura en múltiples tipos de documentos como artículos, tesis, working papers, ponencias e informes técnicos. En la Tabla I, se muestra la ficha de planeación que especifica el objeto, las preguntas de investigación, categorías y ecuaciones de búsqueda desarrolladas. 


\begin{tabular}{|c|c|c|c|}
\hline Objeto de la investigación & \multicolumn{2}{|c|}{ Preguntas de investigación } & Conceptos principales \\
\hline $\begin{array}{l}\text { Enfoques, experiencias y } \\
\text { recursos de preservación } \\
\text { digital a partir del modelo } \\
\text { OAIS para repositorios } \\
\text { institucionales. }\end{array}$ & \multicolumn{2}{|c|}{$\begin{array}{l}\text { ¿Cómo ha sido la implementación del modelo OAIS } \\
\text { en repositorios digitales institucionales para la } \\
\text { preservación digital? } \\
\text { ¿Cuáles instituciones académicas o investigativas han } \\
\text { documentado sus estrategias? } \\
\text { ¿Qué factores conceptuales y aplicados se consideran } \\
\text { importantes para la preservación digital en repositorios } \\
\text { institucionales? }\end{array}$} & $\begin{array}{l}\text { Repositorio digital } \\
\text { Repositorio institucional } \\
\text { Repositorio académico } \\
\text { Repositorio universitario } \\
\text { Preservación digital } \\
\text { Modelo OAIS }\end{array}$ \\
\hline \multicolumn{4}{|c|}{ Ecuaciones de búsqueda } \\
\hline \multicolumn{2}{|c|}{ 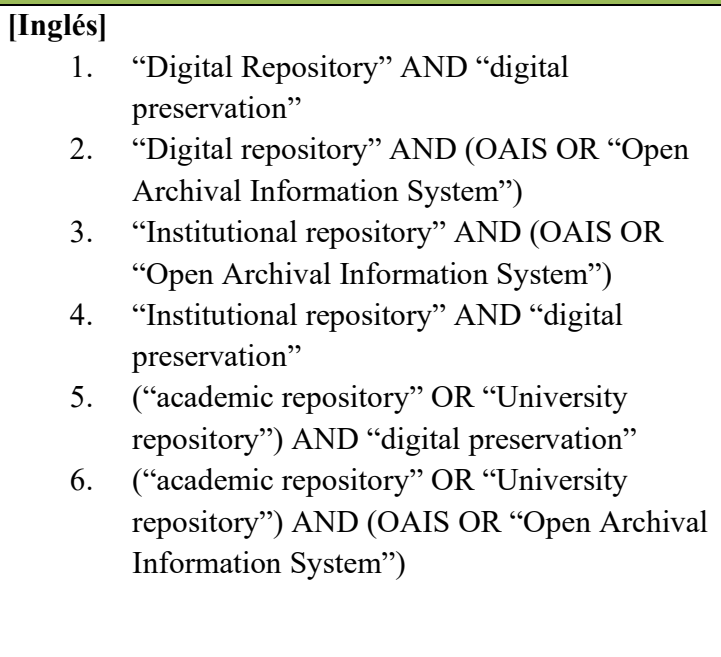 } & $\begin{array}{l}\text { 1. "Repositorio di } \\
\text { digital" } \\
\text { 2. "Repositorio di } \\
\text { "Sistema Abier } \\
\text { Información") } \\
\text { 3. "Repositorio in } \\
\text { "Sistema Abier } \\
\text { Información") } \\
\text { 4. "Repositorio in } \\
\text { "preservación d } \\
\text { 5. ("repositorio ac } \\
\text { universitario") } \\
\text { 6. ("repositorio ac } \\
\text { universitario") } \\
\text { Abierto de Arcl }\end{array}$ & $\begin{array}{l}\text { ital" AND "Preservación } \\
\text { ital" AND (OAIS OR } \\
\text { de Archivo de } \\
\text { titucional" AND (OAIS OR } \\
\text { de Archivo de } \\
\text { titucional" AND } \\
\text { gital" } \\
\text { démico" OR "repositorio } \\
\text { ND "preservación digital" } \\
\text { démico" OR "repositorio } \\
\text { ND (OAIS OR "Sistema } \\
\text { lvo de Información") }\end{array}$ \\
\hline
\end{tabular}

Tabla I. Ficha de planeación de búsqueda de información. Fuente: elaboración propia.

Como criterios de inclusión/exclusión, se tuvieron en cuenta elementos generales de calidad y citación, se analizaron documentos publicados entre 2009 y 2016, considerando aquellos con un nivel de citación igual o mayor a uno, esto teniendo en cuenta que la citación se asocia con criterios de calidad e impacto científico. Respecto a los documentos publicados entre 2017 y 2020, no se tuvo en cuenta este criterio debido a que pueden considerarse de reciente publicación. Se consideró importante que los documentos tuviesen completamente identificado al (los) autor (es) con datos institucionales claramente definidos; para el caso de los libros, se identificaron los datos de la editorial. En cuanto a los working papers, principalmente se eligieron aquellos con información institucional claramente definida. Es importante destacar que solo se consideraron para la investigación aquellos documentos con acceso al texto completo.

A partir de la búsqueda de información, se recuperaron 185 registros. De estos, se seleccionaron 50 asociados al uso del modelo OAIS en repositorios institucionales, en la Figura 1 se puede evidenciar el proceso de depuración de los documentos. Para el análisis de la información, se consideraron categorías emergentes a partir del análisis de los textos, para ello también se tuvieron en cuenta las orientaciones de Neuendorf (2017), Elo y Kyngäs (2008) y Cisterna Cabrera (2005); se construyó una tabla de revisión de experiencias y una categorización general a partir de los procesos del modelo OAIS, modelos de gestión, arquitecturas y sistemas y/o software de repositorios y preservación digital. 


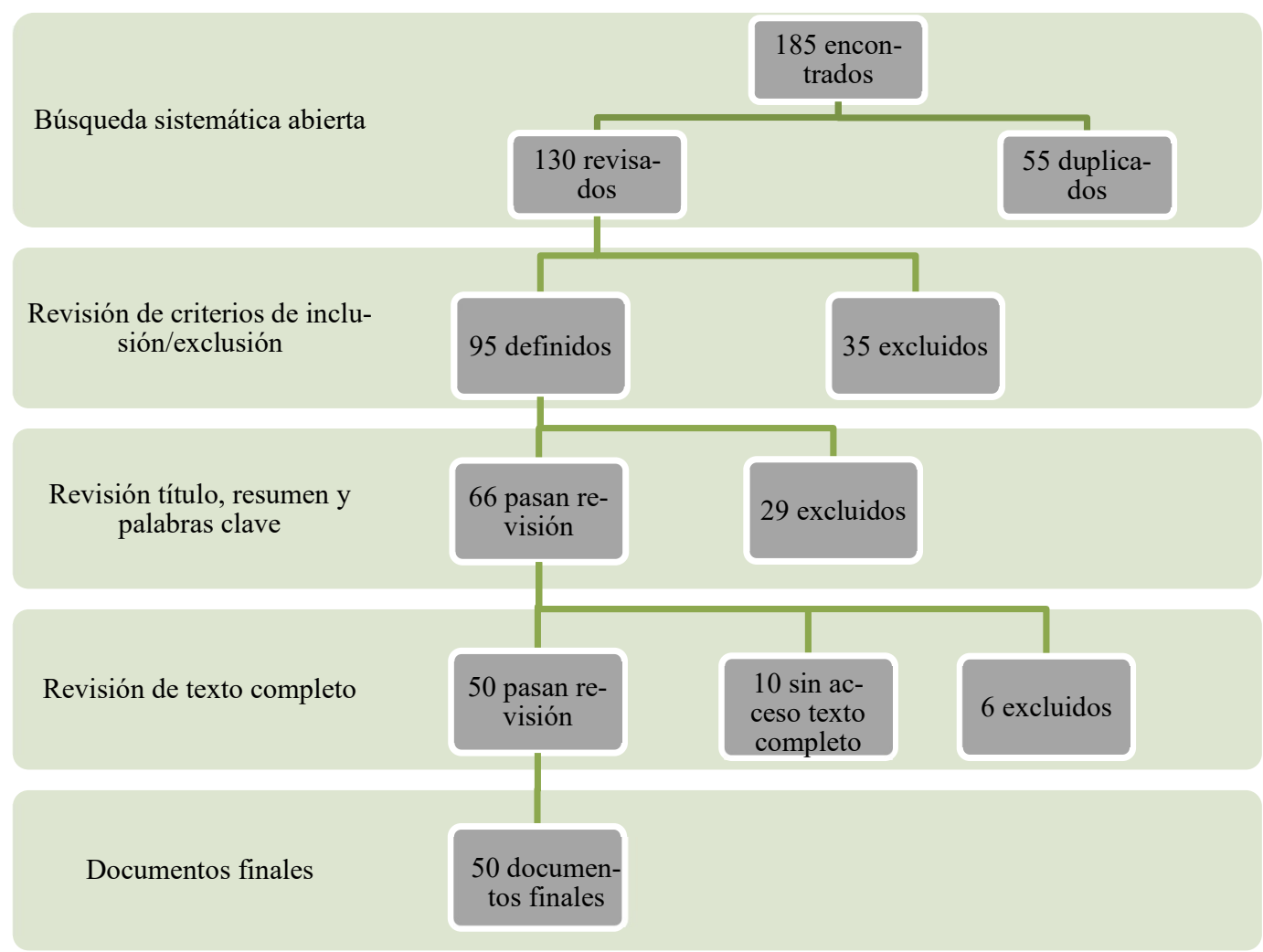

Figura 1. Proceso de selección de documentos para el análisis. Fuente: elaboración propia.

\section{ACCESO ABIERTO Y REPOSITORIOS INSTITUCIONALES}

El movimiento Acceso Abierto surge de la voluntad de científicos y académicos del mundo para poner a libre disposición del público la producción académica de universidades y demás instituciones de investigación, en un acto de responsabilidad social y buen uso de las facilidades que ofrecen el desarrollo de la Internet y la digitalización de contenidos. Gracias a este movimiento, que se guía por iniciativas tales como la Budapest Open Access Initiative (BOAI, 2002), la Declaración de Bethesda (2003), y la Declaración de Berlín (Max Planck Society, 2003), cualquier usuario puede leer, descargar, copiar, distribuir, imprimir, buscar o añadir un enlace al texto completo de esa producción, rastrearla para su indización, incorporarla como datos en un software, o utilizarla para cualquier otro propósito que sea legal, sin barreras financieras, legales o técnicas, aparte de las que son inseparables del acceso mismo a Internet (BOAI, 2002).

En ese contexto, se entiende el Acceso Abierto como:

Una fuente amplia de conocimiento humano y patrimonio cultural aprobada por la comunidad académica que incluye, entre otros, los resultados de la investigación cientifica original, datos primarios y metadatos, materiales, fuentes, representaciones digitales de materiales gráficos y pictóricos, y materiales eruditos en multimedia. (Max Planck Society, 2003).

Una de las modalidades del movimiento Acceso Abierto es la denominada Vía Verde, cuya máxima expresión son los repositorios digitales, ya sean institucionales o temáticos. Los primeros pueden ser considerados como una entidad que recopila, administra y difunde materiales producidos en una institución (Chapman, Reynolds y Shreeves, 2009), mientras que los temáticos se interesan en los productos de un campo científico. Técnicamente, el repositorio es un sistema informático que integra un conjunto de servicios especialmente diseñados para incorporar, reunir, preservar, consultar y dar soporte a la gestión y difusión de los recursos digitales creados por la propia institución o disciplina temática, a través de una interfaz o portal web, mediante una adecuada clasificación de sus recursos a través de metadatos (Lynch, 2003). Además, estos repositorios se caracterizan por su naturaleza digital, por definirse institucionalmente y por ser eruditos, acumulativos, perpetuos, de libre acceso e interoperables (Crow, 2002).

SPARC plantea que un RI es "un archivo digital del producto intelectual creado por la facultad, el personal de investigación y los estudiantes de una institución y accesible para los usuarios finales dentro y fuera de la institución, con pocas o ninguna barrera de acceso" (Crow, 2002, p. 5). En este sentido, también establece que un "repositorio 
institucional podría ser más estrechamente definido como un componente de una iniciativa institucional más integral, que abarque prácticamente todos los activos digitales de una institución" (Crow, 2002, p. 5). Por lo anterior, los repositorios se convierten en una herramienta fundamental para la memoria institucional, por lo que no solo debe centrarse en el almacenamiento y acceso a los objetos digitales, sino en una estructura que amplía su espectro hacia la gestión de información y preservación digital a largo plazo.

No obstante, la forma como los repositorios incorporan la producción científica o académica varía según la política de cada institución, que determina la modalidad del depósito, de acuerdo con criterios de selección previamente definidos por las instituciones. En términos generales, la política del depósito busca establecer que el contenido sea archivado por su respectivo creador, por el propietario o por una tercera parte en su nombre (Alonso Arévalo et al., 2008), en las modalidades de autoarchivo voluntario, autoarchivo obligatorio o depósito delegado.

Esto pone de manifiesto, la gestión de flujos de información de cara a la optimización de la recuperación de información para el libre acceso a la información, pero también la necesidad de definir estrategias oportunas para la optimización de la captura, la organización y la preservación de la información digital. Básicamente, sin una buena estrategia y plan de preservación, no se podrá garantizar el acceso en el tiempo. Por ello, el modelo de referencia OAIS, se constituye como un estándar necesario para garantizar la salvaguarda de la memoria institucional.

\section{MODELO DE REFERENCIA OPEN ARCHIVAL INFORMATION SYSTEM -OAIS-}

El Modelo de Referencia Sistema de Información de Archivo Abierto -OAIS- (CCSDS, 2012), "es una norma ISO (International Standard Organization), desarrollada originalmente por el Consultative Committee for Space Data Systems (CCSDS), que define los procesos necesarios para preservar y acceder a los objetos de información de forma efectiva, a largo plazo y establece un lenguaje común que los describe” (Cruz Mundet y Díez Carrera, 2016), se califica como una "práctica recomendada", es decir, constituye un recurso descriptivo y destinado a proporcionar una orientación general sobre cómo abordar un problema particular (CCSDS, 2012), y se ajusta al concepto de Repositorio Digital Confiable (TDR, por sus siglas en inglés), cuya misión es "ofrecer acceso fiable en el largo plazo a recursos digitales gestionados a una comunidad designada, ahora y en el futuro” (Research Libraries Group, 2002, p. 5).

La Figura 2 representa el modelo OAIS y sus procesos de Captura, Acceso, Administración, Gestión de datos, Planificación de la preservación y Almacenamiento en archivo. Cada uno de ellos se desarrollará en el siguiente apartado.

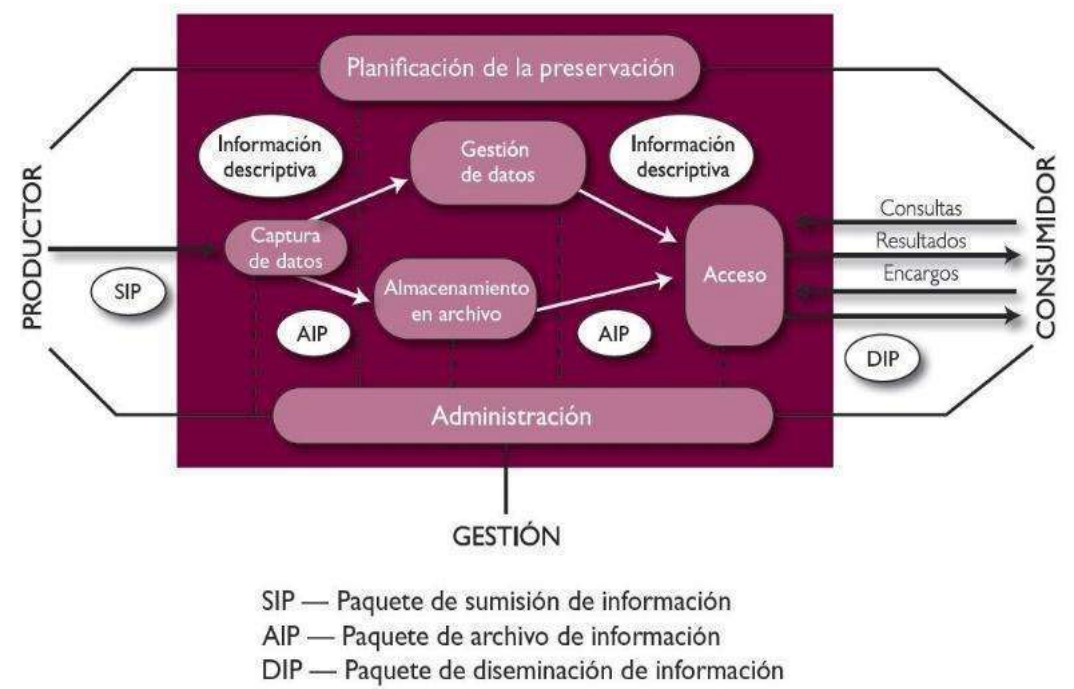

Figura 2. Representación del modelo OAIS. Fuente: IASA (2011).

El modelo OAIS se basa en cinco funcionalidades, tal y como lo muestra Raventós (2010, p. 7) de una forma breve, explicativa y concisa:

Función de adquisición, donde recibe la información de los productores, la prepara para almacenarla y gestionarla en el repositorio; función de gestión de archivo, que define el uso del repositorio, el mantenimiento de paquetes de información de archivo, responsable de asegurar que el contenido que se ha archivado se ha 
hecho de forma adecuada para garantizar su mantenimiento a largo plazo; función de gestión de los datos, que permite describirlos; función de administración que gestiona las operaciones diarias del archivo; función de acceso que ayuda a los usuarios a identificar y obtener la información y permite su consulta.

Para conseguir dar cumplimiento a estas funcionalidades el modelo define tres etapas diferenciadas: la adquisición, la conservación y el acceso. Raventós (2010, p. 7), las explica de la siguiente manera:

La adquisición se puede producir de dos maneras, por entrada directa o después de un proceso de valoración, por lo que el paquete de información puede ser recibido en el formato original o como objeto digital. Su denominación es de Submission Information Package (SIP). Una vez en el sistema el objeto digital se transformará en un objeto digital preservable, el Archival Information Package (AIP), junto con sus metadatos asociados. El nuevo objeto digital se configura a su vez en cuatro paquetes de información sobre el contenido (Content Information), el documento original (Content Data Object), la información de representación (Representation Information) y la información relativa a la conservación (Preservation Description Information). La conservación se puede conseguir gracias al repositorio de archivo y a las estrategias de preservación seleccionadas, con el fin de que el sistema de archivo no quede obsoleto tecnológicamente y se conserven los objetos digitales estables, al igual que las herramientas que permiten su uso. El acceso se consigue a través de la representación del objeto digital (espejo) para facilitar la consulta por parte del usuario, y evitar el riesgo de modificación por uso del objeto original. Su denominación es Dissemination Information Package.

La continua aplicación del modelo OAIS por parte de diferentes instituciones, ha llevado a que sirva como referente para la creación de repositorios digitales confiables, pues además de que lleva asociadas las herramientas tecnológicas específicas para la preservación, se soportan sobre la base de los procesos de un archivo después de su ciclo de transferencia (Sáenz Giraldo, 2017).

La aplicación de este modelo principalmente se ha dado en el marco archivístico. Sin embargo, se deben considerar otros tipos de recursos de información que requieren de orientaciones y tecnologías clave para garantizar la preservación de los documentos digitales en el tiempo. En este caso, los repositorios institucionales, como estrategia bandera del movimiento de acceso abierto a partir de la ruta verde, deben integrar prácticas, protocolos y tecnologías que faciliten la preservación digital de la memoria institucional.

\section{RESULTADOS}

Es necesario comprender que, de cara al mundo digital, a su rápido desarrollo y a los múltiples cambios y peligros que corre la información disponible en los sistemas digitales, se hace necesario contar con sistemas que permitan garantizar el acceso y la integridad de los recursos de información institucional a largo plazo. En este sentido, la preservación digital no debe verse como un conjunto opcional de procesos, tecnologías, protocolos y acciones sino como parte de una estrategia primordial respecto a las buenas prácticas para la gestión de repositorios institucionales.

Se presentan los principales resultados de la revisión realizada sobre los elementos fundamentales de la preservación digital a partir del uso del modelo OAIS en repositorios institucionales. Es importante tener en cuenta que se hace alusión a este modelo precisamente por su amplia difusión y desarrollo en el ámbito de la gestión de información, además de considerarse como un estándar de preservación digital (Cruz Mundet y Díez Carrera, 2016).

La principal característica que se evidencia tras la búsqueda de información es que el modelo OAIS apenas se encuentra en fase de expansión en el uso de repositorios institucionales, esto se manifiesta al ver un número menor de experiencias documentadas. Asimismo, es importante tener en cuenta que este modelo centra su atención en las condiciones de seguridad que se aplican en los repositorios, restringiendo de cierta forma las condiciones de acceso a los documentos originales (CCSDS, 2012), situación que se relaciona directamente con los principios para conservar documentos de archivo y no documentos académicos y científicos, normalmente relacionados con el accionar bibliotecario; en segunda medida, los repositorios institucionales concentran su accionar en el acceso a los documentos a través de esquemas de metadatos normalizados y siguiendo parámetros adecuados; por lo tanto, el modelo generalizado de aplicación en los repositorios es el esquema de metadatos Dublin Core y el protocolo de interoperabilidad OAI-PMH, los cuales responden muy bien para la organización, interoperabilidad y recuperabilidad de la información (Castelli, Manghi y Thanos, 2013) y que, aunque puede facilitar la preservación digital, existen esquemas complementarios que responden de mejor manera a esta necesidad.

Es importante tener en cuenta el tipo de información que se almacena en los repositorios institucionales, tal y como se detalla en la Tabla II; lo cual permite establecer estrategias diferenciadoras entre conceptos que no deben ser contradictorios: el acceso abierto y la preservación digital. Además, para comprender por qué el modelo OAIS puede ser utilizado en repositorios institucionales. 


\begin{tabular}{|l|l|}
\hline \multicolumn{1}{|c|}{ Tipo } & \multicolumn{1}{c|}{ Descripción } \\
\hline Documentos académicos & $\begin{array}{l}\text { Artículos científicos, libros y capítulos de libro, } \\
\text { documentos de trabajo científico, ponencias, entre otros } \\
\text { documentos producto de la actividad académica. }\end{array}$ \\
\hline Tesis y trabajos de grado & $\begin{array}{l}\text { Trabajos de grado de los programas de pregrado y tesis } \\
\text { final de posgrado. }\end{array}$ \\
\hline Documentos patrimoniales & $\begin{array}{l}\text { Documentos históricos con un valor patrimonial para la } \\
\text { institución. }\end{array}$ \\
\hline Documentos producto de la actividad docente & $\begin{array}{l}\text { Documentos de orientación, planeación de las } \\
\text { actividades docentes. }\end{array}$ \\
\hline Documentos producto de las actividades estudiantiles & $\begin{array}{l}\text { Documentos que son producto del desarrollo del ciclo } \\
\text { académico de los estudiantes. }\end{array}$ \\
\hline Objetos de aprendizaje & Recursos digitales con propósitos educativos. \\
\hline Documentos institucionales & $\begin{array}{l}\text { Información institucional de interés para la comunidad } \\
\text { académica. }\end{array}$ \\
\hline
\end{tabular}

Tabla II. Tipo de documentos comunes disponibles en repositorios institucionales. Fuente: elaboración propia.

Por un lado, el acceso abierto, como se ha mencionado anteriormente, manifiesta una premisa donde los repositorios institucionales son una de las rutas que buscan la eliminación de barreras de acceso, difusión y reutilización de recursos resultado de la producción científica y académica. Esto implica un acceso libre y total a los contenidos de este tipo de sistemas.

Por otra parte, la preservación digital debe ser vista mediante:

procesos destinados a garantizar la accesibilidad permanente de los objetos digitales. Para ello, es necesario encontrar las maneras de representar lo que se habia presentado originalmente a los usuarios mediante un conjunto de equipos y programas informáticos que permiten procesar los datos. Para lograrlo, es necesario que la comprensión y la gestión de los objetos digitales se realice considerándolos desde cuatro puntos de vista: como fenómenos físicos, como codificaciones lógicas, como objetos conceptuales comprensibles para el ser humano y como conjuntos de elementos esenciales que deben ser preservados para ofrecer a los futuros usuarios lo esencial del objeto. (Unesco, 2019).

De allí que el modelo OAIS, garantice la aplicación de los elementos, procesos y puntos de vista para representar los contenidos digitales almacenados en cualquier tipo de repositorio.

Considerando entonces, el tipo de información detallado en la Tabla II respecto a los tipos de documentos, se puede expresar que las acciones de preservación digital basadas en un modelo OAIS son de total aplicabilidad para los repositorios institucionales (Raventós, 2010; Yoon y Tibbo, 2011) y de hecho son más que necesarias, pues estos objetos tienen características básicas como: a) son creados por uno o varios autores, b) son almacenados originalmente en el repositorio institucional del que hacen parte el o los autores, c) dan cuenta de la memoria social, científica y académica de la institución, d) son accesibles y, e) se puede extraer información recuperable a través de metadatos. Esto pone de manifiesto la necesidad no solo de promover la visibilidad de la producción científica y académica de las instituciones sino también la de salvaguardar la integridad y acceso a largo plazo.

En general, la literatura evidencia, diversas experiencias relacionadas con la definición de modelos de preservación para repositorios institucionales en múltiples latitudes; algunas de ellas relacionadas con análisis de caso múltiple donde se deja registro de la falta de estrategias y políticas al respecto. No obstante, existen experiencias significativas que pueden verse como lecciones aprendidas frente al tema, además, algunas de ellas refieren a aspectos técnicos como software, modelos y arquitecturas. En la Tabla III se deja registro de los diversos documentos revisados donde el lector puede ubicar el enfoque y nivel de las experiencias relacionadas con repositorios institucionales y preservación digital.

Asimismo, los resultados muestran que más que utilizar una única solución que integre todos los requerimientos, se necesita de sistemas con estrategias, procesos y políticas claras que se puedan desarrollar a partir de la integración de diferentes modelos y software (Palaiologk et al., 2012). Igualmente, la cooperación y los acuerdos internacionales son imperantemente necesarios para definir esquemas de interoperabilidad, uso compartido de recursos y disminución de presupuestos (Silva et al., 2019; Hankinson et al., 2019; Anyaoku, Echedom y Baro, 2019). Esto último es fundamental ante la limitación de recursos financieros y la falta de personal técnico que se ocupe de estos procesos. 


\begin{tabular}{|c|c|}
\hline Categoría & Trabajos \\
\hline $\begin{array}{l}\text { Experiencias generales y análisis de caso sobre políticas } \\
\text { y estrategias de preservación digital. }\end{array}$ & $\begin{array}{l}\text { (Anyaoku, Echedom y Baro, 2019; Beard, 2017; } \\
\text { Chandra y Gokhale, 2012; Cramer y Kott, 2010; da Silva } \\
\text { Júnior y Maria Manuel Borges, 2017; De Giusti y Luján } \\
\text { Villarreal, 2018; Dearborn, Barton y Harmeyer, 2014; } \\
\text { Di Iorio et al., 2014; Díez Carrera, 2012; Dressler, 2017; } \\
\text { Erima, Masai y Wosyanju, 2016; Fernández, 2010; } \\
\text { Gavrilis et al., 2010; Goethals, Frey y Ackerman, 2015; } \\
\text { González y Montero, 2017; Holtkamp et al., 2010; } \\
\text { Ifijeh, 2014; Leija y Térmens, 2017; Manurung, } \\
\text { Distiawan y Putra, 2010; Martin Montull, 2011; } \\
\text { Palaiologk et al., 2012; Pinnick, 2017; REBIUN, 2010; } \\
\text { Rodrigues Viana, Campanha de Carvalho y Flores, } \\
\text { 2014; Rudersdorf, Farrell y Gregory, 2012; Trujillo et } \\
\text { al., 2017; Yance-Yupari, 2019; Beard, 2017; Silva et al., } \\
\text { 2019). }\end{array}$ \\
\hline $\begin{array}{l}\text { Experiencias donde se muestra elementos relacionados } \\
\text { con el software de gestión de repositorios y preservación } \\
\text { digital. }\end{array}$ & $\begin{array}{l}\text { (Boté Vericad y Minguillón, 2012; De Giusti y Luján } \\
\text { Villarreal, 2018; Dearborn, Barton y Harmeyer, 2014; } \\
\text { Hallo Carrasco y de la Fuente, 2010; Jurik et al., 2014; } \\
\text { Leija y Térmens, 2017; Robertson y Borchert, 2014; } \\
\text { Rudersdorf, Farrell y Gregory, 2012; Trujillo et al., } \\
\text { 2017; Xiaolong, Jianmin y Geyond, 2009; Yance- } \\
\text { Yupari, 2019). }\end{array}$ \\
\hline $\begin{array}{l}\text { Experiencias que relacionan modelos y arquitecturas } \\
\text { para la preservación digital en repositorios } \\
\text { institucionales. }\end{array}$ & $\begin{array}{l}\text { (Boté Vericad y Minguillón, 2012; Caplan, Kehoe y } \\
\text { Pawletko, 2010; De Giusti y Luján Villarreal, 2018; De } \\
\text { Giusti, 2014; Di Iorio et al., 2014; Di Iorio y Schaerf, } \\
\text { 2013; Downs y Chen, 2010; Flathers, Kenyon y Gessler, } \\
\text { 2017; Hallo Carrasco y de la Fuente, 2010; Houghton, } \\
\text { 2017; Ivanova, 2011; Jurik et al., 2014; Kowalczyk, } \\
\text { 2015; Palaiologk et al., 2012; Robertson y Borchert, } \\
\text { 2014; Rodrigues Neto, Borges y Roque, 2017; Saini, } \\
\text { 2018; Termens, Ribera y Locher, 2015; Xiaolong, } \\
\text { Jianmin y Geyond, 2009; Hankinson et al., 2019; Azorín } \\
\text { et al., 2020). }\end{array}$ \\
\hline
\end{tabular}

Tabla III. Literatura sobre repositorios institucionales y preservación digital. Fuente: elaboración propia.

\subsection{Experiencias de uso del modelo OAIS en repositorios institucionales}

A partir de esta recopilación de información presentada anteriormente, se muestra algunas experiencias que pueden ayudar a comprender el panorama y la importancia de los procesos, estrategias y tecnología asociadas a la preservación a largo plazo para repositorios digitales, haciendo hincapié en el uso del modelo OAIS. Por tanto, es importante considerar mecanismos para la preservación de la información institucional. En este sentido, la literatura muestra algunas experiencias y reflexiones respecto a los repositorios institucionales que han basado su esquema de planificación, organización y consulta de sus contenidos digitales siguiendo el modelo OAIS.

En la Tabla IV, se muestran las experiencias respecto al diseño de sistemas de preservación digital para repositorios institucionales en Europa y Asia, y se puede ver que existen mayoritariamente experiencias españolas, solo dos casos asiáticos y llama la atención las experiencias a nivel de bloque europeo. Esto no quiere decir que no haya más casos, sino que posiblemente no se han documentado o no se tiene acceso a la información completa. 


\begin{tabular}{|c|c|c|}
\hline País o región & xperiencia y referencia & Descripción \\
\hline India & $\begin{array}{l}\text { Instituto Indio de } \\
\text { Geomagnetismo (IIG) con } \\
\text { el Repositorio institucional } \\
\text { piloto de la AII } \\
\text { (Chandra y Gokhale, 2012) }\end{array}$ & $\begin{array}{l}\text { Usa Dspace como software de repositorio. } \\
\text { Repositorio desagregado para proporcionar servicios de preservación a } \\
\text { pequeños repositorios institucionales. Utiliza SHERPA-OAIS model y } \\
\text { encarga la preservación a una organización externa. } \\
\text { Implementa modelo de preservación de seis etapas, las cuales son: depósito } \\
\text { y generación del paquete de información (SIP), personal del repositorio } \\
\text { realiza control de calidad de metadatos, el SIP se actualiza en el servicio de } \\
\text { preservación, el servicio de preservación genera un paquete de información } \\
\text { de archivo (AIP), este se almacena en un contenedor de un servicio de } \\
\text { terceros (externo), a la vez que se hace copia de seguridad; por último, el } \\
\text { repositorio genera una versión para la consulta pública. } \\
\text { Como estrategia de gobernanza cuenta con una junta asesora con expertos } \\
\text { en preservación. }\end{array}$ \\
\hline Bangladesh & $\begin{array}{l}\text { Universidad de Bangladesh } \\
\text { (Islam et al., 2020) }\end{array}$ & $\begin{array}{l}\text { Este análisis muestra el estado de los repositorios institucionales de } \\
\text { diferentes universidades de Bangladesh. En su mayoría usan software libre } \\
\text { como Dspace. } \\
\text { Se hace énfasis en las actividades de preservación digital de las } \\
\text { universidades; sin embargo, no se ofrecen mayores detalles técnicos y } \\
\text { metodológicos al respecto. }\end{array}$ \\
\hline retaña & $\begin{array}{l}\text { Oxford Common File } \\
\text { Layout (OCFL) } \\
\text { (Hankinson et al., 2019) }\end{array}$ & $\begin{array}{l}\text { Iniciativa compuesta por participantes de Cornell, Stanford, DuraSpace, } \\
\text { Oxford y Emory, la cual permitió el desarrollo de estrategias de } \\
\text { almacenamiento robustas e independientes de la aplicación del repositorio } \\
\text { institucional. } \\
\text { El OCFL soporta la procedencia y el versionado a través de la captura de } \\
\text { un historial de versiones para objetos y la provisión para la implementación } \\
\text { de una auditoría. El control de versiones es eficiente porque el contenido } \\
\text { que no se modifica entre las versiones de los objetos se duplica. El } \\
\text { desarrollo de la iniciativa es un proceso abierto que fomenta la participación } \\
\text { y las aportaciones de la comunidad. Este desarrollo viene siendo probado } \\
\text { en varias instituciones de Gran Bretaña. } \\
\text { Usa el formato Moab (un AIP) diseñado especialmente para repositorios } \\
\text { digitales, y el cual usa XML para conservar la legibilidad humana de los } \\
\text { contenidos y conjunto de metadatos. }\end{array}$ \\
\hline Austria & $\begin{array}{l}\text { Austrian Institute of } \\
\text { Technology } \\
\text { (De Giusti y Luján } \\
\text { Villarreal, 2018; Jurik et } \\
\text { al., 2014) }\end{array}$ & $\begin{array}{l}\text { Usa el software RODA para la preservación digital. } \\
\text { Proyecto denominado: Scalable Preservation Environments (SCAPE). Se } \\
\text { centra en el análisis de formatos de ficheros de repositorios; la descripción } \\
\text { formal de planes y políticas de preservación; la automatización y } \\
\text { vitalización de herramientas y procesos escalables; y el control de calidad } \\
\text { de procesos de preservación. }\end{array}$ \\
\hline Dinamarca & $\begin{array}{l}\text { Red National Danish Bit } \\
\text { Repository } \\
\text { (Leija y Térmens, 2017) }\end{array}$ & $\begin{array}{l}\text { Cuenta con un protocolo y sistema de preservación digital con arquitectura } \\
\text { distribuida y pilares de almacenamiento para que cada institución pueda } \\
\text { gestionar de manera individual o compartida. Como medios de } \\
\text { almacenamiento utiliza unidades de disco magnético, unidades de cinta, } \\
\text { unidades de estado sólido y además, la opción de conectar unidades en la } \\
\text { nube. }\end{array}$ \\
\hline España & $\begin{array}{l}\text { Consorci de Serveis } \\
\text { Universitaris de Catalunya } \\
\text { CSUC } \\
\text { (Leija y Térmens, 2017) }\end{array}$ & $\begin{array}{l}\text { Modelo de red de preservación digital distribuida, participa en el consorcio } \\
\text { MetaArchive Cooperative, basado en el sistema LOCKSS e integra una } \\
\text { capa de coordinación con aplicación web Conspectus. Dentro de este } \\
\text { consorcio el CSUC coordina varias instituciones a partir de un único nodo } \\
\text { de almacenamiento y cooperación, en este sentido su participación es de } \\
\text { miembro colaborativo (collaborative member). }\end{array}$ \\
\hline España & $\begin{array}{l}\text { Universitat Oberta de } \\
\text { Catalunya } \\
\text { (Boté Vericad } \\
\text { Minguillón, 2012) }\end{array}$ & $\begin{array}{l}\text { Repositorio que alberga objetos de aprendizaje de estadística, cuenta con } \\
\text { materiales en diferentes formatos de texto, video y audio. Utiliza el } \\
\text { instrumento de DRAMBORA para el análisis de riesgos. Este modelo les } \\
\text { ha permitido establecer prioridades en el plan de preservación para } \\
\text { determinar la obsolescencia tecnológica. Su principal reto se relaciona con }\end{array}$ \\
\hline
\end{tabular}




\begin{tabular}{|c|c|c|}
\hline País o región & Experiencia y referencia & Descripción \\
\hline & & $\begin{array}{l}\text { la definición de mecanismos semiautomáticos para el etiquetado y } \\
\text { procesamiento de grandes cantidades de objetos de aprendizaje. }\end{array}$ \\
\hline España & $\begin{array}{l}\text { Universidad de Salamanca } \\
\text { (Fernández, 2010) }\end{array}$ & $\begin{array}{l}\text { Usa Dspace como software de repositorio. } \\
\text { El repositorio GREDOS desarrolló una política de preservación para cada } \\
\text { colección digital, cuenta con procedimientos, infraestructura y estrategias } \\
\text { de retención documental para definir los periodos de preservación. } \\
\text { Por último, utiliza estructuras de metadatos Dublin Core, PREMIS, METS } \\
\text { y protocolo OAI-PMH. }\end{array}$ \\
\hline España & $\begin{array}{l}\text { Red de Bibliotecas } \\
\text { Universitarias -REBIUN- } \\
\text { (REBIUN, 2010; Azorín et } \\
\text { al., 2020) }\end{array}$ & $\begin{array}{l}\text { Usa el modelo distribuido elegido por CLOCKSS y se basa en la } \\
\text { arquitectura del software del proyecto LOCKSS: "Lots of copies keep stuff } \\
\text { safe". LOCKSS es un servicio en cual los miembros (principalmente las } \\
\text { grandes bibliotecas de investigación) almacenan en servidores locales las } \\
\text { revistas digitales a las que están suscritas. Se comprueba la integridad de } \\
\text { estos archivos mediante un software "peer-to-peer" que, de forma } \\
\text { automática, detecta y luego corrige los errores identificados en cualquier } \\
\text { servidor de la red de usuarios. } \\
\text { Por otro lado, se destaca la experiencia de REBIUN la elaboración de una } \\
\text { guía para la evaluación de procesos de preservación digital para } \\
\text { repositorios, la cual funciona como instrumento de auditoría interna y por } \\
\text { tanto una buena orientación para cualquier institución. }\end{array}$ \\
\hline Italia & $\begin{array}{l}\text { Sapienza Università di } \\
\text { Roma } \\
\text { (Di Iorio et al., 2014) }\end{array}$ & $\begin{array}{l}\text { Sistema de gestión basado en Fedora Commons (Sapienza) y CINECA. } \\
\text { La Biblioteca Digital de Sapienza (SDL) tiene la infraestructura digital de } \\
\text { conformidad con el modelo funcional OAIS (ISO 14721:2003) y desarrollo } \\
\text { de Servicios compatibles con la Preservación digital a largo plazo (LTDP). } \\
\text { La negociación entre el archivo SDL y las unidades académicas se basan } \\
\text { en un acuerdo, que cubre formalmente todos los recursos enviados al } \\
\text { repositorio digital conservador. El acuerdo establece la adquisición de } \\
\text { software para facilitar los procesos de preservación digital. } \\
\text { El esquema de metadatos fue diseñado para garantizar la conformidad con } \\
\text { el estándar PREMIS, tanto en unidades semánticas como en el nivel de } \\
\text { diccionario de datos. }\end{array}$ \\
\hline $\begin{array}{l}\text { Unión } \\
\text { Europea }\end{array}$ & $\begin{array}{l}\text { Biblioteca Nacional de } \\
\text { España (BNE) } \\
\text { Bibliothèque Nationale de } \\
\text { France (BNF) } \\
\text { British Library (BL) } \\
\text { (Díez Carrera, 2012) }\end{array}$ & $\begin{array}{l}\text { La BNF utiliza sistemas distribuidos de almacenamiento SPAR (Systéme } \\
\text { de Préservation et d'Archivage Repartie), un sistema de almacenamiento } \\
\text { distribuido (LOCKSS) que cumple la norma OAIS (ISO 14721:2003) y } \\
\text { realiza numerosas copias de los objetos digitales, con procedimientos de } \\
\text { vigilancia tecnológica para actualizar formatos, y que permite volver } \\
\text { siempre al formato original ante cualquier contingencia. } \\
\text { La BL utiliza DOM (Digital Object Management) con un control de } \\
\text { integridad para asegurar que no se pierden datos por deterioro o por } \\
\text { catástrofe y seguimiento de versiones y accesibilidad. } \\
\text { Las tres bibliotecas coinciden en el uso de estándares y esquemas como } \\
\text { Marc21, OAI-PMH, Dublin Core, PREMIS y METS. }\end{array}$ \\
\hline $\begin{array}{l}\text { Unión } \\
\text { Europea }\end{array}$ & $\begin{array}{l}\text { European FP7 Research } \\
\text { Programme and aims to } \\
\text { computerize long-term } \\
\text { digital preservation } \\
\text { (Xiaolong; Jianmin y } \\
\text { Geyond, 2009) }\end{array}$ & $\begin{array}{l}\text { Diseño de un sistema basado en agentes inteligentes, recurre a campos } \\
\text { como la inteligencia artificial y la biología. En este sistema los agentes } \\
\text { inteligentes se estructuran en dos niveles: } \\
\text { - En el nivel superior, se definen cuatro tipos de agentes, los cuales son: } \\
\text { agentes de supervisión, agentes de pre injerto, agentes de transferencia } \\
\text { y agentes de ingesta. Estos agentes pueden considerarse los } \\
\text { componentes funcionales del sistema PROTAGE. Son responsables } \\
\text { de diferentes tareas de conservación. } \\
\text { A bajo nivel, existen principalmente dos tipos de agentes, estos son: } \\
\text { agentes de toma de decisiones y agentes de servicios web. Los agentes } \\
\text { de toma de decisiones son responsables de diversas tareas de toma de } \\
\text { decisiones planteadas durante el proceso de preservación digital, } \\
\text { mientras que las tareas de acceso a diversas bases de datos, } \\
\text { repositorios, sistemas de gestión de registros electrónicos (ERMS) y } \\
\text { archivos digitales se asignan a los servicios web. }\end{array}$ \\
\hline
\end{tabular}

Tabla IV. Experiencias de implementación del Modelo OAIS en repositorios institucionales. Fuente: elaboración propia. 
En el caso americano, Tabla V, se evidencian mayoritariamente múltiples experiencias de instituciones estadounidenses. No obstante, llama la atención que existen experiencias en países como Argentina, Brasil y Colombia, lo que demuestra que ha sido un tema de interés para la región latinoamericana, aunque no sea mayoritaria. En cuanto a África, Anyaoku, Echedom y Baro (2019) y Adjei, Mensah y Amoaful (2019) muestran que diversas instituciones cuentan con estrategias de preservación digital a largo plazo, pero tienen dificultades de presupuesto y personal técnico.

\begin{tabular}{|c|c|c|}
\hline País o región & Experiencia y referencia & Descripción \\
\hline EE. UU. & $\begin{array}{l}\text { British Columbia University } \\
\text { (De Giusti y Luján Villarreal, } \\
\text { 2018) }\end{array}$ & $\begin{array}{l}\text { Usa DSpace como software de repositorio. } \\
\text { Repositorio denominado cIRclel, este funciona con DSpace el cual se } \\
\text { encarga del depósito y acceso, mientras que instancias de Archivematica, } \\
\text { en el pipeline, se encargan de la generación del paquete preservable AIP. } \\
\text { El repositorio es el punto de entrada para sus archivos y desde este recibe } \\
\text { las respuestas a las solicitudes de información que ha ingresado. Esta } \\
\text { estructura permite el funcionamiento distribuido. }\end{array}$ \\
\hline EE. UU. & $\begin{array}{l}\text { Bentley Historical Library } \\
\text { (Michigan University) } \\
\text { (De Giusti y Luján Villarreal } \\
\text { 2018) }\end{array}$ & $\begin{array}{l}\text { Usa Dspace como software de repositorio. } \\
\text { Integra funcionalidades de ArchivesSpace y Archivematica y está } \\
\text { orientado a necesidades de preservación y acceso a largo plazo para } \\
\text { objetos nativos digitales. Su estructura se basa en funciones distribuidas. }\end{array}$ \\
\hline EE. UU. & $\begin{array}{l}\text { Purdue University } \\
\text { (Dearborn, Barton y } \\
\text { Harmeyer, 2014) }\end{array}$ & $\begin{array}{l}\text { Utiliza el software HUBzero el cual utiliza esquemas de metadatos como } \\
\text { Dublin Core, METS, MODS, PREMIS y el protocolo de } \\
\text { interoperabilidad y recolección OAI-PMH. } \\
\text { Cuentan con el PURR Team, el cual es un equipo de trabajo que asesora } \\
\text { el desarrollo de procesos, política e infraestructuras para la gestión del } \\
\text { repositorio institucional y la preservación digital de los documentos. Las } \\
\text { estrategias de preservación utilizadas son: reconocimiento de formato, } \\
\text { normalización de metadatos, verificación, migración a formato de } \\
\text { preservación y creación de backup. }\end{array}$ \\
\hline EE. UU. & $\begin{array}{c}\text { Centro de Florida para la } \\
\text { Automatización de } \\
\text { Bibliotecas, Universidad de } \\
\text { Cornell y la Universidad de } \\
\text { Nueva York. } \\
\text { Financiado por: Institute of } \\
\text { Museum and Library } \\
\text { (Instituto de servicios de } \\
\text { museos y bibliotecas) } \\
\text { (Caplan, Kehoe y Pawletko, } \\
\text { 2010) }\end{array}$ & $\begin{array}{l}\text { El objetivo del proyecto es desarrollar, probar y promover un formato de } \\
\text { intercambio estándar para el intercambio de paquetes de información } \\
\text { almacenados entre repositorios de preservación basados en OAIS. } \\
\text { Se busca implementar un modelo de preservación digital distribuida ya } \\
\text { que hay y habrá muchos repositorios de preservación independientes que } \\
\text { almacenan contenido diferente, donde las copias se almacenan en } \\
\text { múltiples ubicaciones. Tiene un único objetivo, diseñar un método } \\
\text { estándar para habilitar el contenido de un repositorio de preservación } \\
\text { basado en OAIS para ser ingerido por otro basado en repositorio OAIS. } \\
\text { Esta experiencia hace parte de un proyecto que busca definir un formato } \\
\text { de paquete de intercambio común, el paquete de intercambio de } \\
\text { repositorio (RXP), y lo deja a los desarrolladores de cada aplicación de } \\
\text { repositorio para desarrollar sus propios mapeos hacia y desde el RXP. }\end{array}$ \\
\hline EE. UU. & $\begin{array}{l}\text { The Stanford Digital } \\
\text { Repository (SDR) } \\
\text { (Cramer y Kott, 2010) }\end{array}$ & $\begin{array}{l}\text { Ha desarrollado un Registro de objetos digitales (DOR) basado en } \\
\text { Fedora para registrar, rastrear y relacionar contenido. Adicionalmente, } \\
\text { facilita los servicios y flujos de trabajo necesarios para acceder y } \\
\text { administrar contenido digital, y prepara los activos para su preservación } \\
\text { y acceso. } \\
\text { El diseño de repositorio se encuentra alineado con el modelo OAIS, sin } \\
\text { embargo, busca implementar una arquitectura y un modelo de datos que } \\
\text { admitirían la ingesta de grandes flujos de contenido, como en Google } \\
\text { Books. } \\
\text { El modelo de datos resta importancia al uso de METS dentro del } \\
\text { repositorio en sí, pero lo respalda como un paquete de difusión cuando } \\
\text { sea necesario. }\end{array}$ \\
\hline EE. UU. & $\begin{array}{l}\text { Harvard Library } \\
\text { (Goethals, Frey y Ackerman, } \\
\text { 2015) }\end{array}$ & $\begin{array}{l}\text { Su enfoque de preservación se concentra en el análisis de los formatos. } \\
\text { Desde este enfoque desarrolló un flujo de trabajo en el que se destacan } \\
\text { la inclusión de un conjunto de criterios para evaluar la obsolescencia del } \\
\text { formato y priorizar su acceso y la creación de perfiles de formato que } \\
\text { ayuda a establecer la estrategia de conservación. Dentro del equipo de } \\
\text { trabajo se incluyeron expertos externos en formatos lo cual permitió } \\
\text { complementar la experiencia interna. }\end{array}$ \\
\hline
\end{tabular}




\begin{tabular}{|c|c|c|}
\hline País o región & Experiencia y referencia & Descripción \\
\hline Colombia & $\begin{array}{l}\text { Universidad del Rosario } \\
\text { Universidad Javeriana } \\
\text { (González y Montero, 2017) }\end{array}$ & $\begin{array}{l}\text { Este caso presenta principalmente recomendaciones para ambas } \\
\text { instituciones respecto al manejo de la preservación digital en los } \\
\text { repositorios institucionales, pues ambas instituciones no tienen ningún } \\
\text { tipo de plan, herramienta o estrategia al respecto. } \\
\text { Entre las principales recomendaciones se cuentan: Crear política de } \\
\text { preservación, realizar auditorías (metodologías de auditoria: ISO } 27000 \text {, } \\
\text { DRAMBORA, Nestor 2, TRAC (ISO/IEC 16363:2012)), evaluar la } \\
\text { opción de acceder y/o contratar servicios de preservación digital como } \\
\text { LOCKSS, CLOCKSS y Pórtico; hacer selección y valoración de } \\
\text { información y vincularse a Redes de RI. }\end{array}$ \\
\hline Brasil & $\begin{array}{l}\text { Cariniana de Brasil } \\
\text { (Leija y Térmens, 2017) } \\
\quad \text { (Silva et al., 2019) }\end{array}$ & $\begin{array}{l}\text { Constituida por varias universidades y centros de investigación, se } \\
\text { presenta como un modelo de preservación digital distribuida sustentado } \\
\text { en el software LOCKS. } \\
\text { El modelo de organización de la red Cariniana distingue tres grupos de } \\
\text { formas de colaboración: en primer lugar, están los colaboradores } \\
\text { integrales, formados por instituciones públicas o privadas que aportan y } \\
\text { comparten infraestructura de almacenamiento y que poseen documentos } \\
\text { digitales en sistemas de gestión electrónica. Luego viene el de las } \\
\text { instituciones colaboradoras, que participan en algún proyecto de } \\
\text { preservación digital y poseen documentos digitales compatibles con los } \\
\text { sistemas de preservación digital, pero que acaban siendo preservados por } \\
\text { los colaboradores integrales. Por último, están los colaboradores } \\
\text { individuales, que son personas que colaboran en proyectos e } \\
\text { investigación en preservación digital. }\end{array}$ \\
\hline Argentina & $\begin{array}{l}\text { Universidad Nacional de La } \\
\text { Plata } \\
\text { (De Giusti y Luján Villarreal, } \\
\text { 2018) }\end{array}$ & $\begin{array}{l}\text { En este repositorio (SEDICI), DSpace se encarga del ingreso y entrega } \\
\text { de contenidos digitales, mientras que Archivematica y ArchivesSpace, } \\
\text { cumplen con actividades de preservación digital. Implementa TRAC } \\
\text { Review Tool para la revisión del proceso. }\end{array}$ \\
\hline Argentina & $\begin{array}{l}\text { Sistema Nacional de } \\
\text { Repositorios Digitales de } \\
\text { Argentina } \\
\text { (Corda, Viñas y Vallefin, } \\
\text { 2020) }\end{array}$ & $\begin{array}{l}\text { Análisis de } 42 \text { instituciones asociadas al Sistema Nacional de } \\
\text { Repositorios Digitales respecto a normas y políticas de preservación } \\
\text { digital. Solamente el } 33 \% \text { de las experiencias cuentan con información } \\
\text { sobre su política institucional al respecto. }\end{array}$ \\
\hline Perú & $\begin{array}{l}\text { Superintendencia Nacional de } \\
\text { Educación Superior } \\
\text { (Yance-Yupari, 2019) }\end{array}$ & $\begin{array}{l}\text { Esta experiencia peruana muestra el análisis de características de los } \\
\text { sistemas de revistas y repositorios de } 19 \text { universidades que no fueron } \\
\text { certificadas por parte de la Superintendencia Nacional de Educación } \\
\text { Superior. En específico, señala los problemas para tener en cuenta de } \\
\text { cara a la preservación digital y los peligros de pérdida de la producción } \\
\text { científica. }\end{array}$ \\
\hline África & $\begin{array}{l}52 \text { bibliotecas universitarias } \\
\text { de África } \\
\text { (Anyaoku, Echedom y Baro, } \\
\text { 2019) }\end{array}$ & $\begin{array}{l}\text { El software mayoritariamente utilizado por las instituciones } \\
\text { universitarias es DSpace. } \\
\text { Las estrategias de preservación implementadas son: migración, } \\
\text { encapsulamiento y actualización. } \\
\text { Participación en programas de preservación digital a través de iniciativas } \\
\text { como Portico y LOCKSS. } \\
\text { El } 58 \% \text { de las instituciones cuentan con políticas de preservación digital } \\
\text { a largo plazo, considerando, incluso, como un elemento prioritario para } \\
\text { los repositorios institucionales. } \\
\text { Sin embargo, esta experiencia no específica las tecnologías y modelos } \\
\text { utilizados, además de considerar como dificultad la falta de presupuesto } \\
\text { y el poco personal con conocimiento técnico. }\end{array}$ \\
\hline África & $\begin{array}{l}\text { Bibliotecas académicas de } \\
\text { Ghana } \\
\text { (Adjei, Mensah y Amoaful, } \\
\text { 2019) }\end{array}$ & $\begin{array}{l}\text { Esta experiencia muestra los resultados de una investigación cualitativa } \\
\text { para las ocho principales bibliotecas académicas de Ghana. } \\
\text { Principalmente muestra la poca existencia de políticas de preservación } \\
\text { digital en estas instituciones; asimismo, se confirma el poco desarrollo } \\
\text { de planes o estrategias asociadas a la preservación digital a largo plazo. }\end{array}$ \\
\hline
\end{tabular}




\begin{tabular}{|l|l|l|}
\hline País o región & Experiencia y referencia & \multicolumn{1}{c|}{ Descripción } \\
\hline & & $\begin{array}{l}\text { Esta experiencia no muestra como tal buenas prácticas o estrategias } \\
\text { claras relacionadas. No obstante, permite dar un panorama de la } \\
\text { situación en este país, el cual no se diferencia de otras latitudes. }\end{array}$ \\
\hline
\end{tabular}

Tabla v. Experiencias de América del Norte, Latinoamérica y África. Fuente: elaboración propia.

A partir de estas experiencias recopiladas, se puede evidenciar que el análisis de información presenta unos elementos clave de cara al desarrollo de proyectos de preservación digital para repositorios institucionales.

1. Las estrategias de colaboración involucran el diseño de un repositorio institucional y otros repositorios se integran al mismo a través de prestación de servicios totales o parciales mediante arquitectura distribuida (Jurik et al., 2014; Chandra y Gokhale, 2012; Leija y Térmens, 2017; Xiaolong, Jianmin y Geyond, 2009; Caplan, 2009).

2. El uso de arquitecturas y sistemas distribuidos garantiza mayor seguridad además de hacer los costos más asequibles para instituciones con poco presupuesto (Leija y Térmens, 2017; Caplan, Kehoe y Pawletko, 2010).

3. Ante la complejidad del proceso y el volumen creciente de información en repositorios institucionales, existe desarrollo de tecnologías que buscan la automatización del proceso de preservación digital. Principalmente se muestran modelos y plataformas basadas en modelos semánticos y/o agentes inteligentes a partir de servicios web. Existen experiencias interesantes como PROTAGE, desarrollado por European FP7 Research Programme (Xiaolong, Jianmin y Geyond, 2009); Sapienza Digital Library (Di Iorio et al., 2014) e INSPIRE, del Laboratorio de Altas Energías (Martin Montull, 2011).

4. DSpace es el software de repositorio más utilizado en repositorios institucionales, pero no es clara la evidencia respecto a sus posibilidades frente a la implementación de modelos de preservación digital como OAIS (Islam et al., 2020; Boté Vericad y Minguillón, 2012; De Giusti y Luján Villarreal, 2018; Caplan, Kehoe y Pawletko, 2010).

5. RODA y Archivematica son los repositorios que aplican completamente el modelo OAIS. Son utilizados en repositorios digitales confiables para documentos de archivo y su aplicación a repositorios institucionales de bibliotecas de investigación es emergente (Trujillo et al., 2017; De Giusti y Luján Villarreal, 2018).

6. El sistema LOCKSS "Lots of copies keep stuff safe", es un servicio que está siendo utilizado por las grandes bibliotecas de investigación para almacenar en servidores locales las revistas digitales a las que están suscritas. Se comprueba la integridad de estos archivos mediante un software "peer-to-peer" que, de forma automática, detecta y luego corrige los errores identificados en cualquier servidor de la red de usuarios (Leija y Térmens, 2017; Robertson y Borchert, 2014).

7. Los esquemas de metadatos utilizados por los repositorios institucionales, independientemente si aplican el modelo OAIS, son: PREMIS, METS y Dublin Core (Di Iorio et al., 2014; Rudersdorf, Farrell y Gregory, 2012; Hallo Carrasco y de la Fuente, 2010; Cramer y Kott, 2010). Esto presenta una oportunidad y reto importante a partir del uso de esquemas de metadatos complejos a partir de la combinación de varios de ellos, asegurando no solo la interoperabilidad y recuperación, sino también la preservación digital. Específicamente, el uso de PREMIS y METS facilitan una tarea que no ha sido tan consciente en los repositorios institucionales.

8. Los formatos utilizados para el almacenamiento de los documentos son: PDF, PDF/A, TIFF (con una resolución mínima de 300 dpi), XML y JPEG (De Giusti y Luján Villarreal, 2018; Robertson y Borchert, 2014). En este elemento, es importante considerar que los repositorios institucionales utilizan formatos estándar y acordes para la preservación, deberá tenerse cuidado con formatos menos comunes y parte de colecciones especiales.

9. Las políticas representan la visión y la orientación en el desarrollo del repositorio, por tanto, no se le debe dar un papel menor, se hace necesario definirlas estratégicamente para definir claramente los procesos y los recursos necesarios (da Silva Júnior y Maria Manuel Borges, 2017; Pinnick, 2017; Dearborn, Barton y Harmeyer, 2014; Dressler, 2017; Anyaoku, Echedom y Baro, 2019; Corda, Viñas y Vallefin, 2020). Respecto a esto, la literatura refiere a las siguientes recomendaciones:

a) Capturar o reunir las colecciones de acuerdo con políticas de desarrollo de colecciones bibliográficas.

b) Conseguir los permisos suficientes con los depositarios para garantizar la conservación de los contenidos.

c) Evaluar constantemente el potencial de la comunidad de usuarios y sus necesidades.

d) Evaluar la colección de metadatos.

e) Recomendar el manejo de ontologías y modelos semánticos.

f) Supervisar constantemente cambios tecnológicos y cambios necesarios en las prácticas de recuperación y preservación de información.

g) Realizar planes de preservación digital (conversión, migración y copias de seguridad).

Todo lo anterior facilita la comprensión de los principales elementos en relación con los requerimientos técnicos, de proceso y a nivel de política para la preservación digital. Ante todo, se hace necesario la comprensión integral del modelo OAIS para su implementación en repositorios institucionales. Considerando estos elementos, Dressler (2017, p. 149) en 
el artículo The state of affairs with digital preservation at ARL member libraries. A survey and analysis of policy, hace un muy buen recuento de los sistemas utilizados en bibliotecas de universidades estadounidenses, que puede ayudar a orientar de mejor manera el trabajo de la preservación digital, al respecto menciona que:

La herramienta técnica que más se mencionó en nueve políticas de preservación digital fue el uso del Programa LOCKSS, una aplicación basada en las bibliotecas de la Universidad de Harvard (2007). La segunda más mencionada es la Cooperativa MetaArchive (una comunidad de colaboración para una solución de preservación digital), presente en cinco políticas. Pórtico y el Digital Preservation Network siguió en un tercio cercano para los servicios basados en suscripción, mencionado en cuatro políticas. Como se reconoció anteriormente, muchas instituciones citaron una combinación de muchas soluciones para la preservación digital y herramientas relacionadas, e incluyen: Preservica, Tessella, Archivematica, Archive-It, Droid, Bag-IT, Bit Curator, Digi Tool, Rosetta, Amazon Glaciar S3 y Hathitrust.

Esto obliga a hacer hincapié en la importancia de trabajar de manera articulada, donde se piense en políticas e infraestructuras interinstitucionales, esto puede ayudar a superar diversas limitaciones que presentan las experiencias entre las que se encuentran el poco presupuesto para la compra de equipos y tecnología, la falta de personal técnico que se encargue de esta tarea y el poco impacto de las políticas. En sí, el trabajo de las redes locales, nacionales y regionales puede representar un muy buen camino para enfrentar los retos que trae la digitalidad y la necesidad de preservación de la memoria institucional a largo plazo.

\section{DISCUSIÓN Y CONCLUSIONES}

La propuesta aquí presentada se basa en el análisis y articulación de dos temas de interés para los repositorios institucionales: el acceso abierto y la preservación digital a través de la aplicación del modelo OAIS (Open Archival Information System). La integración de estos temas asociados a las experiencias internacionales investigadas permite articular acciones de colaboración interinstitucionales mediante sistemas de arquitectura distribuida, automatizar el proceso, diseñar esquemas de metadatos interoperables, conectar sistemas de información para la validación de derechos de autor y aplicar principios y requisitos asociados a la preservación a largo plazo de los objetos digitales. Así, este trabajo muestra diversas experiencias que vislumbran el estado del tema y que permite identificar estructuras alternativas integradoras, lo que también lo convierte en un recurso orientador para el desarrollo de soluciones tecnológicas y de gestión para el diseño de RI.

El movimiento denominado Vía Verde, tiene unos retos que enfrentar en el marco de las realidades tecnológicas que involucran el acceso y la preservación a largo plazo de la memoria científica. En este sentido, los RI tienen un fin claro relacionado con la recopilación y difusión de los materiales producidos por las instituciones, pero su accionar no debe quedarse únicamente en estos factores, sino que debe avanzar en la consolidación de procesos de preservación digital que garanticen la continuidad de los objetos digitales que conserva, la memoria académica, en procurar de mantenerlos accesibles bajo las condiciones de originalidad con las que fueron almacenados inicialmente.

El modelo OAIS representa una oportunidad para que los RI involucren acciones de preservación digital en su proceso sistémico de recopilación y acceso a los documentos. Las universidades, centros de investigación y bibliotecas académicas que están implementando y desarrollando políticas de acceso abierto a través de la conformación de repositorios institucionales deben incluir el modelo OAIS como parte de su diseño y consolidación; esto en clave de las experiencias mundiales que se han logrado sistematizar en este trabajo, permiten identificar elementos necesarios para tomar como referencia: a) conformación de un equipo interinstitucional de la alta dirección que asuma el compromiso por la preservación de la memoria científica; b) formulación de políticas, programas y planes de preservación; c) uso de arquitecturas y sistemas distribuidos interinstitucionales; d) uso de software libre (ejemplo: DSPACE, RODA, Archivematica) y e) diseño de esquemas de metadatos (PREMIS, METS y Dublin Core).

Siguiendo las experiencias mundiales identificadas, la implementación de procesos sistemáticos para la gestión de RI basados en el modelo OAIS, facilitan y optimizan flujos de depósito automático para la disposición, organización y recuperación de la información académica. De esta forma, los RI se pueden concebir a la luz de cinco condiciones básicas: captura, validación de derechos de autor, control bibliográfico, acceso abierto y preservación a largo plazo; de ahí la necesidad de considerar la perspectiva del modelo OAIS para garantizar su gestión integral.

Finalmente, se puede inferir que los RI basados en el modelo OAIS, permiten caracterizar su gestión siguiendo una secuencia interrelacionada entre el ingreso de la información, el almacenamiento de las publicaciones científicas, el acceso a través de estándares de metadatos normalizadores y las consecuentes acciones de preservación digital que 
incluyen el compromiso, el seguimiento y el control de los objetos digitales, mitigando la pérdida de información digital y mejorando la visibilidad de la producción científica y académica.

\section{Agradecimientos}

Este artículo se deriva del proyecto de investigación “Observatorio de las actividades científicas, tecnológicas y de innovación de la Universidad de Antioquia”, financiado por la Vicerrectoría de Investigación de la Universidad de Antioquia UdeA (Medellín - Colombia) y co-financiado por la Escuela Interamericana de Bibliotecología y el Sistema de Bibliotecas de la misma institución. Se agradece la colaboración de los bibliotecólogos Wbeimar Cardona, Mauricio Zapata y del ingeniero Mario Giraldo.

\section{BIBLIOGRAFÍA}

ADJEI, E.; MENSAH, M. y AMOAFUL, E.A. The story so far-digital preservation in institutional repositories: The case of academic libraries in Ghana. Digital Library Perspectives, 2019, vol. 35, $\mathrm{n}^{\circ}$ 2, p. 80-96. Disponible en: $<$ https://doi.org/10.1108/DLP-12-2018-0039>. [Consulta: 15 de septiembre de 2020]

ALONSO ARÉVALO, J., SUBIRATS I COLL, I., MARTÍNEZ CONDE, M.L. y MERLO VEGA, J.A., 2008. Informe APEI sobre acceso abierto. Gijón: APEI. Disponible en: <http://hdl.handle.net/10760/12507>. [Consulta: 15 de septiembre de 2020]

ANYAOKU, E.N.; ECHEDOM, A.U.N. y BARO, E.E. Digital preservation practices in university libraries: An investigation of institutional repositories in Africa. Digital Library Perspectives, 2019, vol. 35, $\mathrm{n}^{\mathbf{0}}$ 1, p. 41-64. Disponible en: < http://dx.doi.org/10.1108/DLP-10-2017-0041>. [Consulta: 1 de octubre de 2020]

AZORÍN, C. et al. Guía para la evaluación de los procesos de preservación digital en repositorios institucionales de investigación. Madrid: REBIUN, 2020.

BEARD, I. The eBethArké Syriac digital library: a case study. Digital Library Perspectives, 2017, vol. 33, $\mathrm{n}^{\circ} 1$, p. 4047. Disponible en: $<$ https://doi.org/10.1108/DLP-07-2016-0017>. [Consulta: 7 de octubre de 2020]

BOAI. Budapest Open Access Initiative [en línea]. 2002. S.1.: s.n. Disponible en: $<$ https://www.budapestopenaccessinitiative.org/translations/spanish-translation> [Consulta: 12 de mayo de 2020]

BOTÉ VERICAD, J. y MINGUILLÓN, J. Preservation of Learning Objects in Digital Repositories. RUSC. Revista de Universidad y Sociedad del Conocimiento, 2012, vol. 9, $\mathrm{n}^{\mathrm{o}}$ 1, p. 22 . Disponible en: $<$ http://dx.doi.org/10.7238/rusc.v9i1 >. [Consulta: 7 de octubre de 2020]

CAPLAN, P. Entender PREMIS. Washington DC: Library of Congress, 2009. Disponible en: $<$ https://www.loc.gov/standards/premis/UnderstandingPREMIS_espanol.pdf $>$. [Consulta: 7 de octubre de 2020]

CAPLAN, P.; KEHOE, W. y PAWLETKO, J. Towards Interoperable Preservation Repositories: TIPR. The International Journal of Digital Curation, 2010, vol. 5, $\mathrm{n}^{\circ} 1, \mathrm{p} .12$.

CASTELLI, D.; MANGHI, P. y THANOS, C. A vision towards Scientific Communication Infrastructures: On bridging the realms of Research Digital Libraries and Scientific Data Centers. International Journal on Digital Libraries, 2013, vol. 13, no 3-4, p. 155-169. Disponible en: <https://doi.org/10.1007/s00799-013-0106-7>. [Consulta: 7 de octubre de 2020]

CCSDS. Reference Model for an Open Archival Information System (OAIS) [en línea]. Washington DC: NASA, 2012. Disponible en: $<$ https://public.ccsds.org/Pubs/650x0m2.pdf $>$. [Consulta: 7 de octubre de 2020]

CHANDRA, S. y GOKHALE, P.A. Implementing Open Archival Information System Model for Digital Preservation at Indian Institute of Geomagnetism. DESIDOC Journal of Library \& Information Technology, 2012, vol. 32, $\mathrm{n}^{\circ} 4$, p. 327-334. Disponible en: <http://dx.doi.org/10.14429/djlit.32.4.2527>. [Consulta: 22 de octubre de 2020]

CHAPMAN, J.W.; REYNOLDS, D. y SHREEVES, S.A. Repository Metadata: Approaches and Challenges. Cataloging \& Classification Quarterly, 2009, vol. 47, $\mathrm{n}^{\mathrm{o}}$ 3-4, p. 309-325. Disponible en: $<$ http://dx.doi.org/10.1080/01639370902735020>. [Consulta: 22 de octubre de 2020]

CISTERNA CABRERA, F. Categorización y triangulación como procesos de validación del conocimiento en investigación cualitativa. Theoria, 2005, vol. 14, $\mathrm{n}^{\mathrm{o}} 1$, p. 61-71.

CORDA, M.C.; VIÑAS, M. y VALLEFIN, C. Preservar la producción académica digital para el futuro: políticas diseñadas en los repositorios de Argentina. Informatio, 2020, vol. 25, $\mathrm{n}^{\mathrm{o}}$ 2. Disponible en: $<$ https://informatio.fic.edu.uy/index.php/informatio/article/view/259> [Consulta: 4 de febrero de 2021]

CRAMER, T. y KOTT, K. Designing and Implementing Second Generation Digital Preservation Services: A Scalable Model for the Stanford Digital Repository. D-Lib Magazine, 2010, vol. 16, $\mathrm{n}^{\circ}$ 9/10. Disponible en: $<\mathrm{http}$ //dx.doi.org/10.1045/september2010-cramer>. [Consulta: 23 de octubre de 2020]

CROW, R. SPARC. Institutional Repository Checklist \& Resource Guide. Washington: SPARC, 2002.

CRUZ MUNDET, J.R. y DÍEZ CARRERA, C. Sistema de Información de Archivo Abierto (OAIS): luces y sombras de un modelo de referencia. Investigación Bibliotecológica: Archivonomía, Bibliotecología e Información, 2016, 
vol. 30, no 70, p. 221-247. Disponible en: <http://dx.doi.org/10.1016/j.ibbai.2016.10.010>. [Consulta: 7 de octubre de 2020]

DA SILVA JÚNIOR, L.P. y MANUEL BORGES, M. Digital preservation policies of the institutional repositories at Brazilian Federal Universities. The Electronic Library, 2017, vol. 35, $\mathrm{n}^{\mathrm{o}}$ 2, p. 311-321. Disponible en: $<$ http://dx.doi.org/10.1108/EL-09-2015-0170>. [Consulta: 7 de octubre de 2020]

DE GIUSTI, M.R. Una metodología de evaluación de repositorios digitales para asegurar la preservación en el tiempo y el acceso a los contenidos [en línea]. Tesis. 2014. S.1.: Universidad Nacional de La Plata. Disponible en: $<$ http://sedici.unlp.edu.ar/handle/10915/43157> [Consulta: 14 de mayo de 2020]

DE GIUSTI, M.R. y LUJÁN VILLARREAL, G. Revision of different implementations for digital preservation: towards a methodological proposal for preserving and auditing IR reliability. RDBCI: Revista Digital de Biblioteconomia e Ciência da Informação, 2018, vol. 16, $\mathrm{n}^{\mathrm{o}}$ 2, p. 273-292. Disponible en: $<$ http://dx.doi.org/10.20396/rdbci.v16i2.8651589>. [Consulta: 7 de octubre de 2020]

DEARBORN, C.; BARTON, A. y HARMEYER, N. The Purdue University Research Repository: HUBzero customization for dataset publication and digital preservation. Libraries Faculty and Staff Scholarship and Research, $2014, n^{\circ} 62$, p. 13.

DI IORIO, A. y SCHAERF, M. Applicability of Digital Library Descriptive Metadata to the Contemporary Artworks En P. NESI y R. SANTUCCI (eds.). Information Technologies for Performing Arts, Media Access, and Entertainment [en línea]. Berlin, Heidelberg: Springer Berlin Heidelberg, 2013, p. 78-89. Disponible en: $<$ http://link.springer.com/10.1007/978-3-642-40050-6_8> [Consulta: 23 de julio de 2020]

DI IORIO, A. et al. A Digital Infrastructure for Trustworthiness. The Sapienza Digital Library Experience. En T. CATARCI, N. FERRO y A. POGGI (eds.). Bridging Between Cultural Heritage Institutions [en línea]. Berlin, Heidelberg: Springer Berlin Heidelberg, 2014, p. 59-69. Disponible en: <http://link.springer.com/10.1007/978-3642-54347-0 7> [Consulta: 23 de agosto de 2020]

DÍEZ CARRERA $\bar{A}, \mathrm{C}$. La preservación digital en las bibliotecas nacionales de la Unión Europea: un análisis comparativo. Boletín ANABAD, 2012, $\mathrm{n}^{\circ}$ 2, p. 171-178.

DOWNS, R.R. y CHEN, R.S. Designing submission and workflow services for preserving interdisciplinary scientific data. Earth Science Informatics, 2010, vol. 3, no 1-2, p. 101-110. Disponible en: $<$ http://dx.doi.org/10.1007/s12145010-0051-6>. [Consulta: 7 de octubre de 2020]

DRESSLER, V.A. The state of affairs with digital preservation at ARL member libraries: A survey and analysis of policy. Digital Library Perspectives, 2017, vol. 33, n 2 2, p. 137-155. Disponible en: <http://dx.doi.org/10.1108/DLP08-2016-0030>. [Consulta: 7 de octubre de 2020]

ELO, S. y KYNGÄS, H. The qualitative content analysis process. Journal of Advanced Nursing, 2008, vol. 62, $\mathrm{n}^{\circ} 1$, p. 107-115. Disponible en: <http://dx.doi.org/10.1111/j.1365-2648.2007.04569.x>. [Consulta: 7 de octubre de 2020]

ERIMA, J.; MASAI, W. y WOSYANJU, M.G. Preservation of digital research content in academic institutions: A case study of Moi University, Kenya. 2016 IST-Africa Week Conference [en línea]. Durban, South Africa: IEEE, 2016, p. 1-11. Disponible en: <http://dx.doi.org/10.1109/ISTAFRICA.2016.7530620>. [Consulta: 15 de octubre de 2020]

FERNÁNDEZ, T.F. Preservación digital en repositorios institucionales GREDOS. Salamanca: Universidad de Salamanca, 2010.

FLATHERS, E.; KENYON, J. y GESSLER, P.E. A service-based framework for the OAIS model for earth science data management. Earth Science Informatics, 2017, vol. 10, $\mathrm{n}^{\mathrm{o}}$ 3, p. 383-393. Disponible en: $<$ http://dx.doi.org/10.1007/s12145-017-0297-3>. [Consulta: 7 de octubre de 2020]

GAVRILIS, D.; PAPATHEODOROU, C.; CONSTANTOPOULOS, P. y ANGELIS, S. Mopseus - A Digital Library Management System Focused on Preservation. En M. LALMAS, J. JOSE, A. RAUBER, F. SEBASTIANI y I. FROMMHOLZ (eds.). Research and Advanced Technology for Digital Libraries [en línea]. Berlin, Heidelberg: Springer Berlin Heidelberg, 2010, p. 445-448. Disponible en: <http://link.springer.com/10.1007/978-3-642-154645_53> [Consulta: 23 de julio de 2020]

GOETHALS, A.; FREY, F. y ACKERMAN, D., 2015. The Evolving Process to Add Preservation Support for New Formats at Harvard Library. Archiving Conference Proceedings. Los Angeles: Society for Imaging Science and Technology, p. 12-15. Disponible en: $<$ https://www.ingentaconnect.com/content/ist/ac/2015/00002015/00000001/art00004>. [Consulta: 7 de octubre de 2020]

GONZÁLEZ, S.M.C. y MONTERO, Y.A.B. Acciones y gestiones en la preservación de los repositorios digitales de las bibliotecas universitarias: estudio de caso de la Universidad del Rosario y de la Universidad Javeriana en Bogotá. Pregrado. Bogotá: Universidad de La Salle, 2017.

HALLO CARRASCO, M. y DE LA FUENTE, P. Preservación digital en el contexto de repositorios institucionales universitarios. Revista Cubana de Ciencias Informáticas, 2010 vol. 4, nº 3-4, p. 1-15.

HANKINSON, A. et al. The Oxford Common File Layout: A Common Approach to Digital Preservation. Publications, 2019, vol. 7, $\mathrm{n}^{\text {o }}$ 2, p. 39. Disponible en: <https://doi.org/10.3390/publications7020039>. [Consulta: 7 de octubre de 2020] 
HIGGINS, J. et al. Cochrane Handbook for Systematic Reviews of Interventions. Oxford: Wiley, 2019.

HOLTKAMP, H.; MELE, S.; SIMKO, T. y SMITH, T. INSPIRE: Realizing the dream of a global digital library in high-energy Physics. Working paper. Ginebra: CERN. 2010-019, 2010.

HOUGHTON, B. Looking back: A university library's self-assessment against ISO 16363:2012. Alexandria: The Journal of National and International Library and Information Issues, 2017, vol. 27, $\mathrm{n}^{\circ}$ 2, p. 108-119. Disponible en: <http://dx.doi.org/10.1177/0955749017725938>. [Consulta: 7 de octubre de 2020]

IASA. Directrices para la producción y preservación de objetos digitales de audio [en línea]. 2011. S.1.: s.n. Disponible en: <https://www.iasa-web.org/tc04-es/la-produccion-y-preservacion-de-objetos-digitales-de-audio> [Consulta: 14 de mayo de 2020]

IFIJEH, G. Adoption of Digital Preservation Methods for Theses in Nigerian Academic Libraries: Applications and Implications. The Journal of Academic Librarianship, 2014, vol. 40, $\mathrm{n}^{\mathrm{o}}$ 3-4, p. 399-404. Disponible en: $<$ http://dx.doi.org/10.1016/j.acalib.2014.06.008>. [Consulta: 7 de octubre de 2020]

ISLAM, M.; UDDIN MUNSHI, N.; ISLAM, N. y HOSSAIN, A. Digital preservation activities of institutional repositories in some selected public university libraries of Bangladesh: an analytical study. The Eastern Librarian, 2020, vol. 25, no 1, p. 24-40.

DECLARACIÓN DE BETHESDA SOBRE PUBLICACIÓN DE ACCESO ABIERTO. [en línea]. 2003. Disponible en: $<$ https://ictlogy.net/articles/bethesda_es.html> [Consulta: 12 de mayo de 2020]

IVANOVA, K. Content-Based Image Retrieval in Digital Libraries of Art Images Utilizing Colour Semantics. En S. GRADMANN, F. BORRI, C. MEGHINI y H. SCHULDT (eds.). Research and Advanced Technology for Digital Libraries [en línea]. Berlin, Heidelberg: Springer Berlin Heidelberg, 2011, p. 515-518. Disponible en: $<$ http://link.springer.com/10.1007/978-3-642-24469-8_62> [Consulta: 23 de julio de 2020]

JURIK, B.A.; BLEKINGE, A.A.; FERNEKE-NIELSEN, R.B. y MOLDRUP-DALUM, P. Bridging the Gap Between Real World Repositories and Scalable Preservation Environments. IEEE/ACM Joint Conference on Digital Libraries. Londres: IEEE, 2014, p. 8.

KOWALCZYK, S.T. Before the Repository: Defining the Preservation Threats to Research Data in the Lab. Proceedings of the 15th ACM/IEEE-CE on Joint Conference on Digital Libraries - JCDL '15 [en línea]. Knoxville, Tennessee, USA: ACM Press, 2015, p. 215-222. Disponible en: <http://dx.doi.org/10.1145/2756406.2756909>. [Consulta: 7 de octubre de 2020]

LEIJA, D. y TÉRMENS, M. Preservación digital distribuida: lecciones de experiencias internacionales. BiD: textos universitaris de biblioteconomia i documentació, 2017, $\mathrm{n}^{\circ} 39$.

LYNCH, C.A. Institutional Repositories: Essential Infrastructure For Scholarship In The Digital Age. Portal: Libraries and the Academy, 2003, vol. 3, n 2, p. 327-336.

MANURUNG, R.; DISTIAWAN, B. y PUTRA, D.D. Developing an Online Indonesian Corpora Repository. PACLIC 24 Proceedings. 2010.Sendai: Institute of Digital Enhancement of Cognitive Processing, 2010, p. $243-249$. Disponible en: <https://www.aclweb.org/anthology/Y10-1028.pdf $>$. [Consulta: 7 de octubre de 2020]

MARTIN MONTULL, J. INSPIRE: Managing Metadata in a Global Digital Library for High-Energy Physics. En E. GARCÍA-BARRIOCANAL, Z. CEBECI, M.C. OKUR y A. ÖZTÜRK (eds.). Metadata and Semantic Research [en línea]. Berlin, Heidelberg: Springer Berlin Heidelberg, 2011, p. 269-274. Disponible en: $<$ http://link.springer.com/10.1007/978-3-642-24731-6_28> [Consulta: 23 de agosto de 2020]

MAX PLANCK SOCIETY. Berlin Declaration on Open Access to Knowledge in the Sciences and Humanities. 2003. Disponible en: <https://openaccess.mpg.de/Berlin-Declaration> [Consulta: 8 de agosto de 2020]

NEUENDORF, K.A. The content analysis guidebook. Second edition. Los Angeles: SAGE, 2017.

PALAIOLOGK, A.S.; ECONOMIDES, A.A.; TJALSMA, H.D. y SESINK, L.B. An activity-based costing model for long-term preservation and dissemination of digital research data: the case of DANS. International Journal on Digital Libraries, 2012, vol. 12, no 4, p. 195-214. Disponible en: <http://dx.doi.org/10.1007/s00799-012-0092-1>. [Consulta: 7 de octubre de 2020]

PINNICK, J. Exploring digital preservation requirements: a case study from the National Geoscience Data Centre (NGDC). Records Management Journal, 2017, vol. 27, $\mathrm{n}^{\circ} 2$.

RAVENTÓS, P. Repositorios digitales: aplicación del modelo OAIS y los esquemas de metadatos a la conservación del patrimonio documental archivístico. El Futuro de la memoria: el patrimonio archivístico digital. 2010. Galicia: Archivo de Galicia, p. 256-276.

REBIUN. Informe del objetivo: Guía de recursos para la preservación digital. S.1.: REBIUN, 2010.

RESEARCH LIBRARIES GROUP. Trusted Digital Repositories: Attributes and Responsibilities [en línea]. S.1.: OCLC, 2002. Disponible en: <https://www.oclc.org/content/dam/research/activities/trustedrep/repositories.pdf $>$ [Consulta: 14 de mayo de 2020]

ROBERTSON, W.C. y BORCHERT, C.A. Preserving Content from Your Institutional Repository. The Serials Librarian, 2014, vol. 66, no 1-4, p. 278-288. Disponible en: <http://dx.doi.org/10.1080/0361526X.2014.881209>. [Consulta: 7 de octubre de 2020] 
RODRIGUES NETO, A.J.; BORGES, M.M. y ROQUE, L. Preliminary Study About the Applicability of a ServiceOriented Architecture in the Oais Model Implementation. Proceedings of the 5th International Conference on Technological Ecosystems for Enhancing Multiculturality - TEEM 2017 [en línea]. Cádiz, Spain: ACM Press, 2017, p. 1-5. Disponible en: <http://dx.doi.org/10.1145/3144826.3145381>. [Consulta: 7 de octubre de 2020]

RODRIGUES VIANA, G.F.; CAMPANHA DE CARVALHO, T. y FLORES, D. Preservação da memória institucional relativa a projetos universitários através do sistema aberto de arquivamento de informação (SAAI). Ibersid, 2014, $\mathrm{n}^{\circ}$ 8, p. 103-108.

RUDERSDORF, A.; FARRELL, D. y GREGORY, L. Electronic records processing: it's a CINCH! Proceedings of the 12th ACM/IEEE-CS joint conference on Digital Libraries - JCDL '12 [en línea]. Washington, DC, USA: ACM Press, 2012, p. 375. Disponible en: <http://dx.doi.org/10.1145/2232817.2232898>. [Consulta: 7 de octubre de 2020]

SÁENZ GIRALDO, A. Preservación digital en Colombia desde la perspectiva archivística: análisis de políticas, niveles de cumplimiento y un esquema de preservación digital para Colombia. Trabajo fin de máster. Barcelona: Universitat Autònoma de Barcelona, 2017.

SAINI, O.P. Understanding the Role of Institutional Repository in Digital Preservation in Academic Libraries: A Review of Literature. Library Philosophy and Practice, 2018, vol. 7, n 2, p. 1-14.

SILVA, M.P. de B. e; MOURA, R.K.G. de; SIEBRA, S. de A. y PINTO, V.B. Contribuições da Rede Cariniana para a preservação digital nos repositórios digitais institucionais. Informação em Pauta, 2019, vol. 4, nº especial 2, p. 99116. Disponible en: <http://dx.doi.org/10.32810/2525-3468.ip.v4iEspecial.2019.42607.99-116>. [Consulta: 7 de octubre de 2020]

TERMENS, M.; RIBERA, M. y LOCHER, A. An analysis of file format control in institutional repositories. Library Hi Tech, 2015, vol. 33, no 2, p. 162-174. Disponible en: <http://dx.doi.org/10.1108/LHT-10-2014-0098>. [Consulta: 7 de octubre de 2020]

TRUJILLO, S. et al. Archivematica outside the box: Piloting a common approach to digital preservation at the Five College Libraries. Digital Library Perspectives, 2017, vol. 33, $\mathrm{n}^{\circ}$ 2, p. 117-127. Disponible en: $<$ http://dx.doi.org/10.1108/DLP-08-2016-0037>. [Consulta: 7 de octubre de 2020]

UNESCO. Noción de preservación digital. UNESCO, 2019 [en línea]. Disponible en: $<$ https://es.unesco.org/themes/information-preservation/digital-heritage/concept-digital-preservation> [Consulta: 9 de mayo de 2020]

VICENTE, R.S.; MELERO, R.M. y ABADAL, E. Indicadores para la evaluación de repositorios institucionales de acceso abierto. Anales de Documentación, 2014, vol. 17, $\mathrm{n}^{\mathrm{o}} 2$. Disponible en: $<$ http://dx.doi.org/10.6018/analesdoc.17.2.190821>. [Consulta: 1 de octubre de 2020]

XIAOLONG, J.; JIANMIN, J. y GEYOND, M. Towards computerized digital preservation based on intelligent agents and web services. Proceedings of the Fifth International Conference on Web Information Systems and Technologies [en línea]. Lisbon, Portugal: SciTePress - Science and and Technology Publications, 2009, p. 276-281. Disponible en: <http://dx.doi.org/10.5220/0001826802760281>. [Consulta: 7 de octubre de 2020]

YANCE-YUPARI, V. ¿La producción científica puede desaparecer? Problemas de preservación digital en las universidades peruanas. Cultura, 2019, vol. 33, p. 347-362. Disponible en: $<$ https://doi.org/10.24265/cultura.2019.v33.19>. [Consulta: 7 de octubre de 2020]

YOON, A. y TIBBO, H. Examination of Data Deposit Practices in Repositories with the OAIS Model. IASSIST Quarterly, 2011, vol. 35, n 4, p. 6-13. 